This item was submitted to Loughborough's Research Repository by the author.

Items in Figshare are protected by copyright, with all rights reserved, unless otherwise indicated.

\title{
Data envelopment analysis models of investment funds
}

PLEASE CITE THE PUBLISHED VERSION

http://dx.doi.org/10.1016/j.ejor.2011.08.019

PUBLISHER

(c) Elsevier

VERSION

SMUR (Submitted Manuscript Under Review)

LICENCE

CC BY-NC-ND 4.0

REPOSITORY RECORD

Lamb, John D., and Kai-Hong Tee. 2019. "Data Envelopment Analysis Models of Investment Funds”. figshare. https://hdl.handle.net/2134/14797. 
This item was submitted to Loughborough's Institutional Repository (https://dspace.lboro.ac.uk/) by the author and is made available under the following Creative Commons Licence conditions.

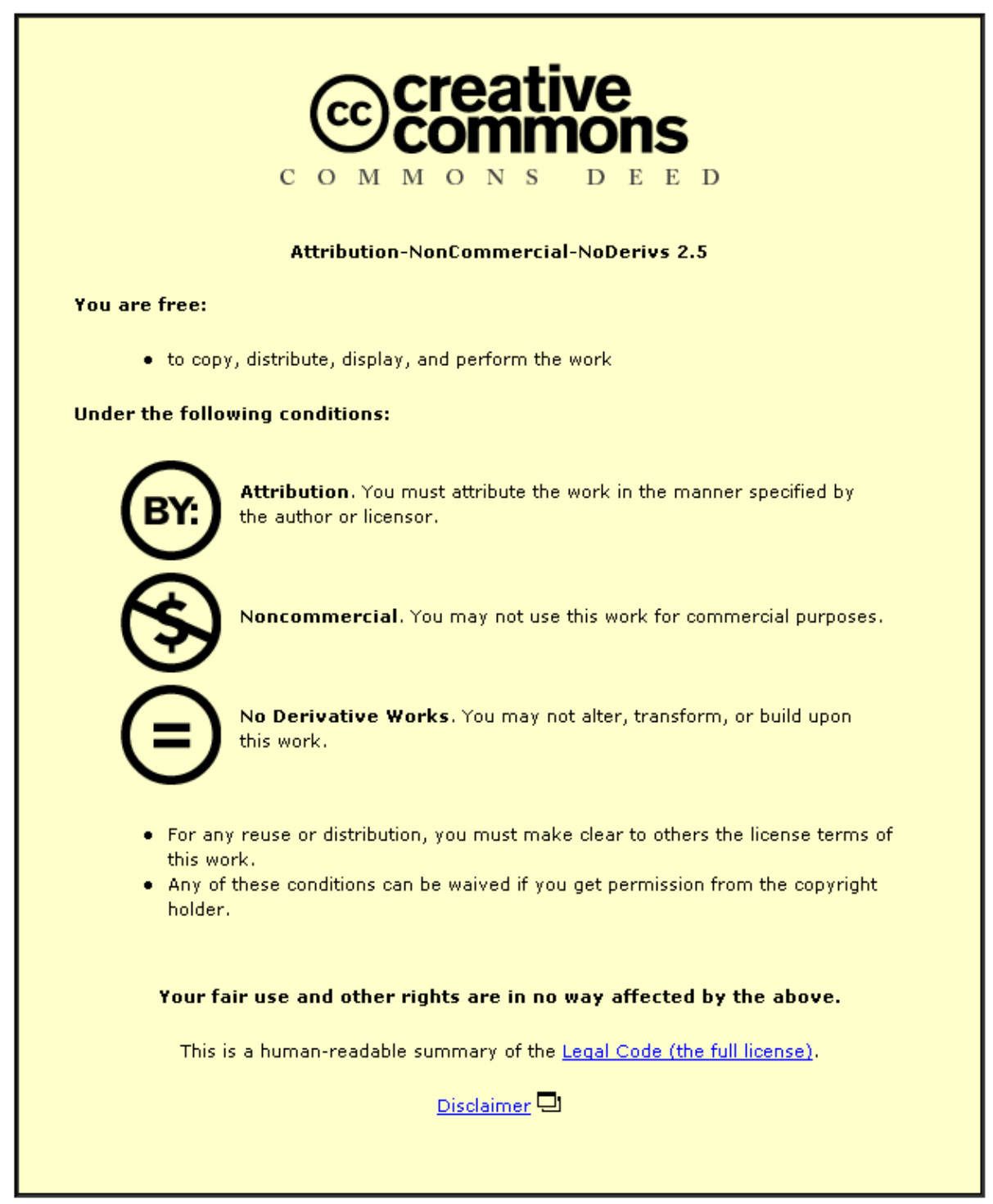

For the full text of this licence, please go to: http://creativecommons.org/licenses/by-nc-nd/2.5/ 


\title{
Data envelopment analysis models of investment funds
}

\author{
John D. Lamb ${ }^{\mathrm{a}, *}$, Kai-Hong Tee ${ }^{\mathrm{b}}$ \\ ${ }^{a}$ University of Aberdeen Business School, Aberdeen AB24 3QY \\ ${ }^{b}$ Loughborough University Business School, Loughborough LE11 3TU
}

\begin{abstract}
This paper develops theory missing in the sizable literature that uses data envelopment analysis to construct return: risk ratios for investment funds. It explores the production possibility set of the investment funds to identify an appropriate form of returns to scale. It discusses what risk and return measures can justifiably be combined and how to deal with negative risks, and identifies suitable sets of measures. It identifies the problems of failing to deal with diversification and develops an iterative approximation procedure to deal with it. It identifies relationships between diversification, coherent measures of risk and stochastic dominance. It shows how the iterative procedure makes a practical difference using monthly returns of 30 hedge funds over the same time period. It discusses possible shortcomings of the procedure and offers directions for future research.
\end{abstract}

Keywords: data envelopment analysis, investment fund, diversification, coherent risk measure, returns to scale, stochastic dominance

\section{Introduction}

Data envelopment analysis (DEA) estimates the technical efficiency of several decision-making units (DMUs) given several inputs and several outputs. Typically the efficiency may be written as

$$
\frac{\text { weighted sum of outputs }}{\text { weighted sum of inputs }} \text {. }
$$

*Corresponding author: Tel: +44 (0) 1224 274362; fax: +44 (0) 1224273843.

Email addresses: J.D.Lamb@abdn.ac.uk (John D. Lamb), K.Tee@lboro.ac.uk (Kai-Hong Tee) 
Authors such as Murthi et al. (1997) and Gregoriou and Zhu (2005) observe the following analogy. If we use risk in place of input and return in place of output in expression (1), we get a ratio that generalises return-risk ratios such as the Sharpe (Sharpe, 1966), Calmar and Sortino (Lhabitant, 2004) ratios, used to compare investment funds. The benefit of DEA is that it lets us account for different investor attitudes to risk and return.

The published literature on DEA for investment funds is not theoretically justified. In using the analogy between output-input and return-risk ratios it usually makes an implicit assumption that fund returns are perfectly correlated. And it often ignores the need for comparable measures of risk and return. We investigate theoretically when and how DEA may be used to compare investment funds, and develop a new method that models diversification directly. To do this we discuss four important issues.

1. DEA depends on technical assumptions of free disposability and convexity. The literature does not discuss whether these reasonably hold when modelling investment funds. We show that, in general, they do not.

2. Originally DEA (Charnes et al., 1978) assumed constant returns to scale (CRS) but later allowed for variable returns to scale (vRs) (Banker et al., 1984). Both are used for investment funds (Gregoriou and Zhu, 2005), but the analogy between output-input and risk-return suggests neither and many authors use one or other with limited or no justification. For example, Gregoriou et al. (2005a) argue for a vRs model only because it compensates for what Section 3 identifies as noncommensurable measures.

3. The literature allows for different investor preferences by combining measures such as mean, median, standard deviation (Gregoriou et al., 2005a), lower and upper semivariance and semiskewness (Gregoriou et al., 2005b), skewness (Wilkens and Zhu, 2001), excess kurtosis (NguyenThi-Thanh, 2006), time horizons (Galagadera and Silvapulle, 2002), percentage of periods with negative returns, skewness (Wilkens and Zhu, 2001), value at risk, conditional value at risk (Chen and Lin, 2006), downside absolute standard deviation, weighted absolute deviation from quantile, and tail value at risk (Lozano and Gutiérrez, 2008). Eling (2006) reviews the measures used and concludes there is no single standard choice. Two problems arise. Some measures use different units from others. And some may take negative values. 
4. We need to deal with diversification because a combination of funds may be more efficient than any individual fund. Basso and Funari (2001); Sengupta (2003) account for diversification indirectly using market $\beta$ (Alexander, 2001) as a risk measure. Morey and Morey (1999); Joro and Na (2006); Briec and Kerstens (2009); Lozano and Gutiérrez (2007) account for it directly using nonlinear versions of DEA. And Lozano and Gutiérrez (2008) account for it directly in linear DEA, limited to models with only one input and one output.

We resolve these issues as follows. Section 2 discusses the assumptions needed for a DEA model of investment funds and how to handle returns to scale. Section 3 introduces commensurability as a way of choosing a set of risk and return measures that can justifiably be combined. Section 4 explains coherent measures of risk and how to satisfy the assumption of convexity and model diversification through a series of linear approximations to an ideal nonlinear model. Section 5 illustrates our method using monthly returns from 30 hedge funds over the same time period. Section 6 discusses possible shortcomings of our method and some future directions for research.

Coherent measures of risk come up again and again in our discussion. They relate to investor preference because they help model stochastic dominance. They allow us to account for distributional shape and also to use multiple risk measures. They also relate closely to what we need in order to deal with diversification. The best-known coherent measure of risk is conditional value at risk (CVaR), which is based on value at risk (VaR). VaR and CVaR are families of risk measures, allowing us to account for many different investor preferences and dsutributional shapes. VaR is the maximum loss that investors might suffer over a time horizon at a specified confidence level. CVaR is the expected loss conditional on this loss exceeding VaR. It is sometimes called expected shortfall, tail conditional expectation, conditional loss or tail loss (Jorion, 2007). Acerbi and Tasche (2002) note some subtle differences among these terms, which we can ignore for continuous distribution functions, and show that CVaR is a coherent measure of risk. Acerbi (2007) shows how to estimate CVar from a sample. We use this in Section 5.

\section{Background}

This section describes DEA models for investment funds and the assumptions needed for them and shows an NRS model is appropriate. 
A DEA model compares $n$ DMUs using $m$ input and $s$ output measures. An investment fund (or fund for short) produces returns that we can measure at regular time intervals. Section 5 uses examples with average monthly returns. We assume the regular returns are realisations of a real random variable and ignore, for now, the possibility that the random variable is a function of time. We suppose we have funds described by random variables $f_{1}, \ldots, f_{n}$. We define a random variable $\sum_{j=1}^{n} \lambda_{j} f_{j}$ with $\lambda_{j} \geq 0(j=1, \ldots, n)$ and $\sum_{j=1}^{n} \lambda_{j} \leq 1$ to be a portfolio on $f_{1}, \ldots, f_{n}$ and we define the portfolio possibility set $\mathcal{F}$ to be the set of all such portfolios. Then a measure is a function $g: \mathcal{F} \rightarrow \mathbb{R}$. Since a measure is a population statistic, we invariably must estimate it with a sample statistic. We call the sample statistic also a measure and use the same notation for both, distinguishing only when the difference is unclear. We classify some measures as return measures and some as risk measures. For example, the mean value is a return measure and the standard deviation a risk measure. Later we describe other risk and return measures and what properties they might have. We suppose we have risk measures $x_{1}, \ldots, x_{m}$ and return measures $y_{1}, \ldots, y_{s}$. We write $x_{i j}=x_{i}\left(f_{j}\right)$, $y_{r j}=y_{r}\left(f_{j}\right), \mathbf{x}_{j}=\left(x_{1 j}, \ldots, x_{m j}\right)$ and $\mathbf{y}_{j}=\left(y_{1 j}, \ldots, y_{s j}\right)(i=1, \ldots, m$, $r=1 \ldots, s, j=1, \ldots, n)$ so we can describe DEA compactly.

The (input-oriented) DEA model is this. For each DMU $o \in\{1, \ldots, n\}$ choose $\mathbf{u}_{o} \in \mathbb{R}^{s}, \mathbf{v}_{o} \in \mathbb{R}^{m}$, and $\alpha \in \mathbb{R}$ to

maximise

$$
\phi_{o}=\frac{\alpha+\mathbf{y}_{o} \cdot \mathbf{u}_{o}}{\mathbf{x}_{o} \cdot \mathbf{v}_{o}}
$$

subject to

$$
\frac{\alpha+\mathbf{y}_{j} \cdot \mathbf{u}_{o}}{\mathbf{x}_{j} \cdot \mathbf{v}_{o}} \leq 1, \quad(j=1, \ldots, n)
$$

and

$$
\mathbf{u}_{o} \geq \mathbf{0}, \mathbf{v}_{o} \geq \mathbf{0}
$$

Note that we solve (2)-(4) separately for each $o$. The efficiency of DMU $o$ is $\phi_{o}$, which must be in $[0,1]$ provided $\mathbf{x}_{j}>\mathbf{0}$ and $\mathbf{y}_{j} \geq \mathbf{0}$ for $j=1, \ldots, n$. We set $\alpha=0$ for a CRS model and do not constrain it for a VRS model. We use an input-oriented model because funds are usually compared using return-risk ratios like expression (2). Gregoriou and Zhu (2005) show an 
output-oriented model is possible. A slacks-based model (Tone, 2001) is also possible, though we know of none used to model funds.

Model (2)-(4) is sometimes called a ratio model and is usually recast as a linear program. We present the dual form. Gregoriou and Zhu (2005) give details. This form lets us discuss the assumptions needed for DEA, relate them to portfolios and the assumptions needed to model funds, describe the efficient frontier, and discuss what form of returns to scale is appropriate. We find the CRS DEA efficiencies from the following (dual) linear programmes for each $o=1, \ldots, n$. Choose $\phi_{o}, \lambda_{1 o}, \ldots, \lambda_{n o}$ to

minimise

$\phi_{o}$

subject to

$$
\begin{aligned}
& \sum_{j=1}^{n} y_{r j} \lambda_{j o} \geq y_{r o}, \quad(r=1, \ldots, s) \\
& \sum_{j=1}^{n} x_{i j} \lambda_{j o} \leq x_{i o} \phi_{o}, \quad(i=1, \ldots, m)
\end{aligned}
$$

and

$$
\lambda_{j o} \geq 0 \quad(j=1, \ldots, n) .
$$

To get the VRS model we add the following constraint to (5)-(8).

$$
\sum_{j=1}^{n} \lambda_{j o}=1 .
$$

Following Cazals et al. (2002), we define a VRS production possibility set as a set $\Psi=\left\{(\mathbf{x}, \mathbf{y}) \in \mathbb{R}_{+}^{m} \times \mathbb{R}_{+}^{s}\right\}$ satisfying

Free disposability: (see, for example, (Shephard, 1970)): if $(\mathbf{x}, \mathbf{y}) \in \Psi$, then $\left(\mathbf{x}^{\prime}, \mathbf{y}^{\prime}\right) \in \Psi$ whenever $\mathbf{x}^{\prime} \geq \mathbf{x}$ and $\mathbf{y}^{\prime} \leq \mathbf{y}$.

Convexity: if $(\mathbf{x}, \mathbf{y}),\left(\mathbf{x}^{\prime}, \mathbf{y}^{\prime}\right) \in \Psi$ then $\left(t \mathbf{x}+(1-t) \mathbf{x}^{\prime}, t \mathbf{y}+(1-t) \mathbf{y}^{\prime}\right) \in \Psi$ for $t \in(0,1)$. 
$\Psi$ is the set of $(\mathbf{x}, \mathbf{y})$ such that it is possible to produce $\mathbf{y}$ from $\mathbf{x}$. Free disposability and convexity are usually assumed to be reasonable for DEA models in economics, though Bogetoft (1996); Cazals et al. (2002) discuss cases where convexity is questionable. The input-oriented efficient frontier of $\Psi$ is the set $\left\{(\mathbf{x}, \mathbf{y}) \in \Psi:\left(\mathbf{x}^{\prime}, \mathbf{y}\right) \notin \Psi\right.$ for $\left.\mathbf{x}^{\prime}<\mathbf{x}\right\}$. It is straightforward to show that if $\lambda_{j 1}, \ldots, \lambda_{j n}, \phi_{o}$ solves the VRS DEA model, then $\sum_{j=1}^{n}\left(\mathbf{x}_{j}, \mathbf{y}_{j}\right) \lambda_{j}$ is on the efficient frontier of the smallest production possibility set containing all the DMUs and so $\phi_{o}$ is a measure of the efficiency of DMU $o$. It is also straightforward to check that we get the corresponding result for the CRS model if we expand the definition of $\Psi$ so that $(t \mathbf{x}, t \mathbf{y}) \in \Psi$ whenever $(\mathbf{x}, \mathbf{y}) \in$ $\Psi$ and $t>0$.

Figure 1 about here.

Figure 1 shows the mean (return) and standard deviation (risk) in the monthly returns of 30 funds described in Section 5. The darker shaded area is the production possibility set for the VRS model. Its frontier is shown as a line on the left of this area that is dashed then solid. It represents the possibility, common in economic models, that the marginal return on output eventually decreases as input increases. The total shaded area is the production possibility set for the CRS model. Its frontier is shown as a line on the left of this area that is solid then dashed.

We know of no literature that checks if the standard assumptions about production possibility sets and returns to scale are valid for models of funds. They are not. There is a minor problem with free disposability. Suppose we use $\operatorname{VaR}_{0.2}$ and $\operatorname{VaR}_{0.05}$ as risk measures and have $\operatorname{VaR}_{0.2}(f)=0.1$ and $\operatorname{VaR}_{0.05}(f)=0.2$ for fund $f$. Then the free disposability assumption suggests an investment $f^{\prime}$ with $\operatorname{VaR}_{0.2}\left(f^{\prime}\right)=0.3$, $\operatorname{VaR}_{0.05}\left(f^{\prime}\right)=0.2$ is possible because it exceeds $f$ in one risk measure. But $\operatorname{VaR}_{0.05} \geq \operatorname{VaR}_{0.2}$ by definition. This does not invalidate DEA because the efficiencies do not depend on free disposability. So we assume from here on that free disposability holds for any production possibility set we consider.

There is a much bigger problem with convexity. Suppose $\mathbf{x}(g)$ is the risk measure and $\mathbf{y}(g)$ the return measure for each fund $g$. And suppose $f$ and $f^{\prime}$ are funds. Then, for $t \in(0,1)$ we can assume that $\left(\mathbf{x}\left(t f+(1-t) f^{\prime}\right), \mathbf{y}(t f+\right.$ $\left.\left.(1-t) f^{\prime}\right)\right) \in \Psi$ because $t f+(1-t) f^{\prime}$ is a possible portfolio. But the equalities

$$
\begin{aligned}
& t \mathbf{x}(f)+(1-t) \mathbf{x}\left(f^{\prime}\right)=\mathbf{x}\left(t f+(1-t) f^{\prime}\right), \\
& t \mathbf{y}(f)+(1-t) \mathbf{y}\left(f^{\prime}\right)=\mathbf{y}\left(t f+(1-t) f^{\prime}\right)
\end{aligned}
$$


only hold for perfectly correlated funds for all measures we know of other than mean value. So convexity is at best an approximation. Section 4 develops a method to deal with this approximation.

We now consider returns to scale in models of funds. A vRs model is implausible because it allows for a minimum level of risk before a positive return is possible. A CRS model is implausible because it allows returns greater than any portfolio can produce. The portfolio possibility set allows us to choose a portfolio that has some proportion uninvested: that is, invested with zero risk and zero return. Assuming equalities (10) are reasonable, this is equivalent to defining the production possibility set $\Psi$ to satisfy

NRS convexity: if $(\mathbf{x}, \mathbf{y}),\left(\mathbf{x}^{\prime}, \mathbf{y}^{\prime}\right) \in \Psi$ then $\left(t \mathbf{x}+u \mathbf{x}^{\prime}, t \mathbf{y}+u \mathbf{y}^{\prime}\right) \in \Psi$ for $t$, $u \geq 0, t+u \leq 1$.

The solid line in Figure 1 shows the efficient frontier of the smallest production possibility set containing all 30 funds and satisfying this. The corresponding DEA model is (5)-(8) with the extra constraint

$$
\sum_{j=1}^{n} \lambda_{j o} \leq 1
$$

We call it a nonincreasing returns to scale (NRS) model. Cooper et al. (2007) describe it as a possible relaxation of constraint (9) and Färe and Lovell (1994) discuss how NRS efficiencies can be used to classify returns to scale in a general DEA model. Joro and Na (2006) use NRS with limited explanation in a nonlinear mean-variance-skewness model. It is straightforward to check, using linear programming duality that the NRS model is equivalent to (2)-(4) with $\alpha \leq 0$. We consider only NRS models from here on.

\section{Commensurable sets of measures}

We call a set of inputs and outputs commensurable if each input or output is measured in a positive constant multiple of some common unit. This section argues that any DEA model should ideally use only commensurable sets of inputs and outputs. Later it argues that an input-oriented model of funds should use nonnegative risk measures and shows there are reasonable sets of measures that are commensurable and contain only nonnegative risk measures. 
We use commensurable measures for two reasons. First, as (Lovell and Pastor, 1995) note, the efficiency, given by equation (2), should be dimensionless. Second, we want the efficiencies to be units invariant: multiplying the fund returns by a positive constant should not change the efficiencies. Lovell and Pastor (1995) note that most models, including all the models we discuss, have a weaker form of units invariance: multiplying the values of any input or output by a positive constant should not change the efficiencies.

In practice, the measures in a commensurable set usually include the mean and so have the same units as the funds' returns. Some published models use measures such as variance, semivariance, skewness and kurtosis that are not commensurable with the mean return (Wilkens and Zhu, 2001; Gregoriou et al., 2005b; Nguyen-Thi-Thanh, 2006). There are several plausible explanations. First, funds returns are usually expressed as proportions and so it may not be immediately obvious that the efficiencies are not dimensionless. Second, DEA models are often expressed in dual form (like model (5)-(9)) in which the units of the efficiencies are less obvious. Third, some nonlinear models (Morey and Morey, 1999; Joro and Na, 2006; Briec and Kerstens, 2009) must use noncommensurable measures to be practically solvable. For example, Morey and Morey (1999) use mean, variance and covariance as measures in models with quadratic constraints.

Initially DEA did not allow negative values for inputs and outputs. This is usually reasonable for economic models but less so for models of funds. Even the mean return can be negative, as Figure 1 illustrates. If $y_{\text {ro }} \leq 0$ for some $r$ and $o$ then $\phi_{o}=0, \lambda_{1 o}=\cdots=\lambda_{n o}=0$ satisfies constraints (6)-(8), (11). But $\phi_{o} \geq 0$ to satisfy constraints (7) provided $x_{i o}>0$ for some $i$. So negative return measures are largely unproblematic, and a NRS model with positive inputs and outputs unrestricted in sign is equivalent to one with positive inputs in which any negative output value is replaced by zero.

Negative or zero risk measures are more problematic. If $x_{i o}<0$ for some $i$ it is possible that $\phi_{o}>1$. And if $\mathbf{x}_{o}=\mathbf{0}, \phi_{o}=-\infty$. So we need positive risk measures for a reasonable definition of efficiency. However, to allow riskfree investments, ideally we would like at least the possibility that the risk measure could be zero. One approach to the problem of nonpositive input values is to add a constant to each value. If doing this does not change the efficiencies, we can call the input translation invariant. While some DEA models exhibit (at least limited) translation invariance (Lovell and Pastor, 1995), it is easy to check that the inputs in the NRS model are not translation invariant. So ideally we should avoid this approach. Another approach deals 
with negative values by measuring efficiency relative to an ideal point (Silva Portela et al., 2004). We eschew it because we have found no way to make it compatible with the production possibility set assumptions for a set of funds. Instead we define the complete DEA model as follows. Let $\mathbf{x}_{j} \geq \mathbf{0}$ and $\mathbf{y}_{j} \geq \mathbf{0}$ for $j=1, \ldots, n$. If $\mathbf{x}_{o}>\mathbf{0}$ define $\phi_{o}$ as in model (5)-(8), (11); otherwise put $\phi_{o}=1$ or 0 according as $\mathbf{y}_{o} \geq \mathbf{0}$ or $\mathbf{y}_{o}<\mathbf{0}$. The complete model has the desirable property that a risk-free fund is efficient whenever the value of at least one return measure is nonnegative. We wish to establish further the consistency in the definition of $\phi_{o}$. To do this we first show how the NRS and VRS models are related.

Suppose that $\mathbf{x}_{j}>\mathbf{0}$ and $\mathbf{y}_{j}>\mathbf{0}$ for $j=1, \ldots, n-1$, and $\mathbf{x}_{n}=\mathbf{y}_{n}=\mathbf{0}$. Then for $j=1, \ldots, n-1$, we can write the programmes of the VRS model (5)(9) as minimise $\phi_{o}$ subject to $\sum_{j=1}^{n-1} y_{r j} \lambda_{j o} \geq y_{r o}(r=1, \ldots, s), \sum_{j=1}^{n-1} x_{r j} \lambda_{j o} \leq$ $x_{i o} \phi_{o}(i=1, \ldots, m), \sum_{j=1}^{n-1} \lambda_{j o} \leq 1$, and $\lambda_{j o} \geq 0(j=1, \ldots, n-1)$. This is just the NRS model with DMUs $1, \ldots, n-1$. It follows that we can find the efficiencies of an NRS model by solving all but one of the programmes of the VRS model we get by adding an extra DMU with all inputs and outputs equal to zero.

We now investigate the consistency of the complete DEA model. This matters because Section 4 creates expanded models that typically include a notional fund $o$ with $\mathbf{x}_{o}=\mathbf{0}$ and $\mathbf{y}_{o}>\mathbf{0}$. Ideally we would like to show that $\phi_{o}$, considered as a function of $\mathbf{x}_{1}, \ldots, \mathbf{x}_{n}, \mathbf{y}_{1}, \ldots, \mathbf{y}_{n}$, is continuous. Although continuity of efficiency measures is desirable (Russell, 1990), Scheel and Scholtes (2003) show for the VRS model that, although $\phi_{o}$ is continuous almost everywhere, it may have discontinuities on the frontier and at points where $x_{i j}=0$. We also deliberately allow a discontinuity at $\mathbf{y}_{o}=\mathbf{0}$ because it is unlikely that a rational investor would consider a fund with no risk and negative return to be efficient. The following result shows $\phi_{o}$ is continuous at $\mathbf{x}_{o}=\mathbf{0}$ if $\mathbf{y}_{o} \geq \mathbf{0}$ and $x_{i j}>0$ for $j \neq o$ and for all $i$. We write $\phi_{o}$ for the efficiency of DMU $o$ in the complete VRS model and $\phi_{o}^{\prime}$ for the efficiency of the oth DMU in the complete VRS model with DMUs given by inputs $\mathbf{x}_{j}^{\prime}$ and outputs $\mathbf{y}_{j}^{\prime}(j=1, \ldots, n)$. We use $\mathbf{x}, \mathbf{y}$ as a shorthand for $\mathbf{x}_{1}, \ldots, \mathbf{x}_{n}, \mathbf{y}_{1}, \ldots, \mathbf{x}_{n}$ and, for $\varepsilon>0$, write $N_{\varepsilon}(\mathbf{x}, \mathbf{y})$ for $\left\{\mathbf{x}^{\prime}, \mathbf{y}^{\prime}:\left\|\left(\mathbf{x}^{\prime}, \mathbf{y}^{\prime}\right)-(\mathbf{x}, \mathbf{y})\right\|_{2}<\varepsilon\right\}$, the $\varepsilon$-neighbourhood of $(\mathbf{x}, \mathbf{y})$.

Theorem 1. Suppose $\mathbf{x}_{o}=\mathbf{0}, \mathbf{y}_{o} \geq \mathbf{0}$ and $x_{i j}>0$ for all $i$ and for all $j \neq o$. Then $\phi_{o}^{\prime}=\phi_{o}$ for all $\left(\mathbf{x}^{\prime}, \mathbf{y}^{\prime}\right) \geq \mathbf{0}$ sufficiently close to $(\mathbf{x}, \mathbf{y})$.

Proof. By definition $\phi_{o}=1$. Put $\varepsilon=0.5 \min \left\{x_{i j}: i \in\{1, \ldots, m\}, j \in\right.$ 
$\{1, \ldots, n\} \backslash\{o\}\}>0$, and choose $\left(\mathbf{x}^{\prime}, \mathbf{y}^{\prime}\right) \in N_{\varepsilon}(\mathbf{x}, \mathbf{y})$. Then $x_{i j}^{\prime} \geq x_{i o}^{\prime}$ for all $i$ and $j$. If $\mathbf{x}_{o}^{\prime}=\mathbf{0}$ then $\phi_{o}^{\prime}=1$ by definition, and so $\phi_{o}^{\prime}=\phi_{o}$. Otherwise, choose $i$ such that $x_{i o}^{\prime}>0$. Then constraint (7) gives

$$
\phi_{o}^{\prime} x_{i o}^{\prime} \geq \sum_{j=1}^{n} x_{i j}^{\prime} \lambda_{j o} \geq \sum_{j=1}^{n} \lambda_{j o} x_{i o}^{\prime}=x_{i o}^{\prime} \quad \text { (using constraint (9)). }
$$

Since $x_{i o}^{\prime}>0$, it follows that $\phi_{o}^{\prime} \geq 1$. The feasible solution $\phi_{o}^{\prime}=1, \lambda_{o o}=1$, $\lambda_{j o}=0(j \neq o)$ implies $\phi_{o}^{\prime} \leq 1$, and so $\phi_{o}^{\prime}=1=\phi_{o}$ as required.

It follows from the relation between the NRS and VRS models that the result holds mutatis mutandis for the complete NRS model.

From here on we consider only DEA models of investment funds and use only models based on the complete NRS model. It remains to show there are commensurable sets of measures suitable for a complete NRS model. Return measures are straightforward since they can be negative: the mean, upper quantiles and upper tail means are in the same units as the data. Risk measures need to be nonnegative. The standard deviation, absolute deviation and lower semideviation are all reasonable. And VaR and CVaR are measured in the same units as the data and are usually positive. Theorem 3 shows how we can deal with negative values.

\section{Diversification, convexity, and coherent measures of risk}

We wish to compare investment funds to the risks and returns available from any portfolio composed from them. To do this we must consider diversification because diversification may let us reduce the risk of an investment without reducing its return, violating one of the basic assumptions of standard DEA.

This section starts with a mathematical programming model that handles diversification. The model is not usually practically solvable. The section then shows this model is a convex programming problem if its measures have properties similar to the well-known properties of coherent measures of risk. The section continues by presenting an iterative procedure that approximates the solution of the convex programming problem. It finishes with some technical results on convergence and properties of the models discussed. 


\section{Diversification: an impractical model}

Section 2 defines the production possibility set $\Psi$ for a set of funds as the set of values of $\left(\left(x_{1}(f), \ldots, x_{m}(f)\right),\left(y_{1}(f), \ldots, y_{s}(f)\right)\right)$ such that $f$ is in the portfolio possibility set $\mathcal{F}$. If the measures are nonnegative and form a commensurable set, the efficiency $\phi_{o}$ with respect to $\Psi$ is given by the complete NRS model if $\mathbf{x}_{o}=\mathbf{0}$ and by the following mathematical program otherwise. Choose $\phi_{o}, \lambda_{1 o}, \ldots, \lambda_{n o}$ to

minimise $\phi_{o}$ subject to

$$
\begin{aligned}
& y_{r}\left(\sum_{j=1}^{n} \lambda_{j o} f_{j}\right) \geq y_{r}\left(f_{o}\right), \quad(r=1, \ldots, s) \\
& x_{i}\left(\sum_{j=1}^{n} \lambda_{j o} f_{j}\right) \leq x_{i}\left(f_{o}\right) \phi_{o}, \quad(i=1, \ldots, m) \\
& \sum_{j=1}^{n} \lambda_{j o} \leq 1,
\end{aligned}
$$

and

$$
\lambda_{j o} \geq 0 \quad(j=1, \ldots, n) .
$$

We call this model the diversification-consistent (input-oriented complete NRS DEA) model because it compares each fund accurately with all possible portfolios and so deals fully with diversification.

The diversification-consistent model is important because it disposes of the assumption, implicit in conventional DEA, that

$$
\kappa\left(\sum_{j=1}^{n} \lambda_{j} f_{j}\right)=\sum_{j=1}^{n} \lambda_{j} \kappa\left(f_{j}\right)
$$

for each $\kappa \in\left\{x_{1}, \ldots, x_{m}, y_{1}, \ldots, y_{s}\right\}$. This assumption holds if $\kappa$ is the mean value, but is otherwise unlikely to hold. The main problem with the diversification-consistent model is that is not generally solvable by any known practical method.

The model can use any risk and return measures though we restrict our attention to commensurable sets of nonnegative measures for the reasons Section 3 discusses. 
A number of published models, though not in the form of the diversificationconsistent model, deal directly with correlation. Lozano and Gutiérrez (2008, 2007) describe diversification-consistent linear DEA models with precisely one input and one output. They estimate additive metrics of the form $\delta_{\mathrm{r}}+\delta_{\mathrm{d}}$ rather than efficiencies, though the models can easily be adjusted to calculate NRS efficiencies. They achieve linearity by specifying particular estimators (and not just particular measures) for the risk measures that depend on having a vector time series of returns assumed to be stationary and without serial autocorrelation. The sets of measures are commensurable though the estimator may be biased for measures such as CVar (see Acerbi (2007)) if we make the reasonable assumption that the distribution of returns is not discrete. Morey and Morey (1999) describe a DEA models of investment funds that deals with correlation using variance as a risk measure and includes a quadratic constraint. Joro and $\mathrm{Na}$ (2006) extend this to include skewness in a model with cubic constraints. Briec et al. (2004); Briec and Kerstens (2009, 2010) use a directional distance function rather than standard DEA and develop nonlinear programming models to allow higher moments as measures. The main drawback of the published nonlinear models is that they use noncommensurable sets of measures to get solvable mathematical programmes.

Measures that make the diversification-consistent model a convex programming problem

The diversification-consistent model is likely to be more tractable if we can find measures that make it a convex programming problem. And it is a convex programming problem whenever $\Psi$ satisfies the assumptions of free disposability and NRS convexity. It is easy to check that NRS convexity is equivalent to requiring

$$
x_{i}\left(\sum_{j=1}^{n} \lambda_{j} f_{j}\right) \leq \sum_{j=1}^{n} \lambda_{j} x_{i}\left(f_{j}\right) \quad \text { and } \quad y_{r}\left(\sum_{j=1}^{n} \lambda_{j} f_{j}\right) \leq \sum_{j=1}^{n} \lambda_{j} y_{r}\left(f_{j}\right)
$$

for $i=1, \ldots, m$ and $r=1, \ldots, s$ in the diversification-consistent model.

We need measures satisfying inequalities (15). Artzner et al. (1999) call a measure $\kappa: \mathcal{F} \rightarrow \mathbb{R}$ a coherent measure of risk if it satisfies the following four properties. Here $r \in \mathbb{R}$ represents the total rate of return on a reference instrument.

Translation invariance: for $f \in \mathcal{F}$ and $\alpha \in \mathbb{R}^{+}, \kappa(f+\alpha r)=\kappa(f)-\alpha$. 
Subadditivity: for $f, g \in \mathcal{F}, \kappa(f+g) \leq \kappa(f)+\kappa(g)$.

Positive homogeneity: for $\lambda \geq 0$ and $f \in \mathcal{F}, \kappa(\lambda f)=\lambda \kappa(f)$.

Monotonicity: for $f, g \in \mathcal{F}$ with $f \leq g, \kappa(g) \leq \kappa(f)$.

Translation invariance ensures that if we add $r \kappa(f)$ to $f$ we get a random variable with risk measured as zero. Subadditivity ensures a sum of random variables has no greater risk than the sum of the risks of the individual random variables. Positive homogeneity ensures the risk is proportional to the size of investment. And monotonicity ensures that coherent risk measures are consistent with a general stochastic ordering. We define an additional property that is useful for return measures.

Superadditivity: for $f, g \in \mathcal{F}, \kappa(f+g) \geq \kappa(f)+\kappa(g)$.

The following theorem shows how some of these properties are useful. We define a measure to be convexity consistent if it is positively homogeneous and either: (i) a risk measure and subadditive; or (ii) a return measure and superadditive.

Theorem 2. Suppose that each measure in a diversification-consistent model is convexity consistent. Then its production possibility set $\Psi$ satisfies NRS convexity.

Proof. Suppose $\left(\mathbf{x}_{1}, \mathbf{y}_{1}\right),\left(\mathbf{x}_{2}, \mathbf{y}_{2}\right) \in \Psi$ and $t, u \in(0,1)$ with $t+u \leq 1$. Then, for some $f_{1}, f_{2} \in \mathcal{F}$ we have $\mathbf{x}\left(f_{1}\right) \leq \mathbf{x}_{1}, \mathbf{y}\left(f_{1}\right) \geq \mathbf{y}_{1}, \mathbf{x}\left(f_{2}\right) \leq \mathbf{x}_{2}$ and $\mathbf{y}\left(f_{2}\right) \geq \mathbf{y}_{2}$.

Put $\mathbf{x}^{\prime}=\left(x_{1}^{\prime}, \ldots, x_{m}^{\prime}\right)=\mathbf{x}\left(t f_{1}+u f_{2}\right)$ and for $j=1,2$ write $\mathbf{x}_{j}=$ $\left(x_{1 j}, \ldots, x_{m j}\right)$. Then, for $i=1, \ldots, m$,

$$
\begin{aligned}
& x_{i}^{\prime}=x_{i}\left(t f_{1}+u f_{2}\right) \\
& \leq x_{i}\left(t f_{1}\right)+x_{i}\left(u f_{2}\right) \quad \text { (subadditivity) } \\
& =t x_{i}\left(f_{1}\right)+u x_{i}\left(f_{2}\right) \quad \text { (positive homogeneity) } \\
& \leq t x_{i 1}+u x_{i 2} \text {. }
\end{aligned}
$$

Hence $t \mathbf{x}_{1}+u \mathbf{x}_{2} \geq \mathbf{x}^{\prime}$ 
Similarly, put $\mathbf{y}^{\prime}=\left(y_{1}^{\prime}, \ldots, y_{s}^{\prime}\right)=\mathbf{y}\left(t f_{1}+u f_{2}\right)$ and for $j=1,2$ write $\mathbf{y}_{j}=\left(y_{1 j}, \ldots, y_{s j}\right)$. Then, for $r=1, \ldots, s$,

$$
\begin{aligned}
& y_{r}^{\prime}=y_{r}\left(t f_{1}+u f_{2}\right) \\
& \geq y_{r}\left(t f_{1}\right)+y_{r}\left(u f_{2}\right) \quad \text { (superadditivity) } \\
& =t y_{r}\left(f_{1}\right)+u y_{r}\left(f_{2}\right) \quad \text { (positive homogeneity) } \\
& \geq t y_{r 1}+u y_{r 2} \text {. }
\end{aligned}
$$

Hence $t \mathbf{y}_{1}+u \mathbf{y}_{2} \leq \mathbf{y}^{\prime}$.

Since we defined $\mathbf{x}^{\prime}$ and $\mathbf{y}^{\prime}$ so that $\left(\mathbf{x}^{\prime}, \mathbf{y}^{\prime}\right) \in \Psi$, it follows from the free disposability of $\Psi$ that $\left(t \mathbf{x}_{1}+u \mathbf{x}_{2}, t \mathbf{y}_{1}+u \mathbf{y}_{2}\right) \in \Psi$. It follows that $\Psi$ satisfies NRS convexity.

We now show there are practical convexity-consistent measures. It is easy to check that standard deviation is convexity consistent, and it is nonnegative by definition. Theorem 2 shows any nonnegative coherent measure of risk is convexity consistent. Krause (2002) and Acerbi and Tasche (2002) describe a range of coherent risk measures including lower semideviation and $\mathrm{CVaR}_{\alpha}$. We can use several values of $\alpha$ to get several risk measures and so model much of the shape of the distribution. It is easy to check the mean and - CVaR are convexity-consistent return measures. Typically we might use $\mathrm{CVaR}_{\alpha}$ as a risk measure for $\alpha \leq 0.4$ and $-\mathrm{CVaR}_{\alpha}$ as a return measure for $\alpha \geq 0.6$.

Coherent measures of risk may have negative values, but we need nonnegative risk measures. The following result shows we can construct a nonnegative convexity-consistent risk measure from any coherent measure.

Theorem 3. Suppose $\mathcal{F}$ is a class of random variables and $x: \mathcal{F} \rightarrow \mathbb{R} a$ coherent measure of risk. Then $\hat{x}=\max (x, 0)$ is positively homogeneous, subadditive and monotonic.

Proof. Let $f$ and $g \in \mathcal{F}$. Then, for $\lambda \geq 0$,

$$
\hat{x}(\lambda f)=\max (x(\lambda f), 0))=\max (\lambda x(f), 0)=\lambda \hat{x}(f) .
$$

So $\hat{x}$ is positively homogeneous. And

$$
\begin{aligned}
\hat{x}(f+g) & =\max (x(f+g), 0) \leq \max (x(f)+x(g), 0) \\
& \leq \max (x(f), 0)+\max (x(g), 0)=\hat{x}(f)+\hat{x}(g) .
\end{aligned}
$$

So $\hat{x}$ is subadditive. It follows that $\hat{x}$ is convexity consistent. Finally,

$$
\begin{aligned}
f \leq g & \Rightarrow x(g) \leq x(f) \Rightarrow \max (x(g), 0) \leq \max (x(f), 0) \\
& \Rightarrow \hat{x}(g) \leq \hat{x}(f) .
\end{aligned}
$$


So $\hat{x}$ is monotonic.

Note that the newly-constructed measure is not, in general, translation invariant. The main benefit of translation invariance is that it lets us to include a reference instrument in the measure. This does not matter when we can include it in the model.

\section{A practical iterative solution method}

The production possibility set of the complete NRS model is the smallest set containing $\left(\mathbf{x}_{j}, \mathbf{y}_{j}\right)(j=1, \ldots, n)$ and satisfying free disposability and NRS convexity. So it is contained in the production possibility set $\Psi$ of the diversification-consistent model whenever $\Psi$ is convex. This happens whenever all the measures are convexity consistent. Then the efficiencies of the complete NRS model estimate the efficiencies of the diversificationconsistent model, but may do so poorly.

We want to improve the estimates of the efficiencies of the diversificationconsistent model. The following model helps. It is no more than the complete NRS model with some extra funds, which are portfolios composed of the original funds. We call these portfolios notional funds.

For $k=1, \ldots, \hat{n}$ and $(j=1, \ldots, n)$ choose $\mu_{j k} \geq 0$ so that $\sum_{j=1}^{n} \mu_{j k} \leq 1$. Write $\hat{f}_{k}=\sum_{j=1}^{n} \mu_{j k} f_{j}$. Put $\hat{x}_{i j}=x_{i}\left(\hat{f}_{j}\right),(i=1, \ldots, m, j=1, \ldots, \hat{n})$ and $\hat{y}_{r j}=y_{r}\left(\hat{f}_{j}\right),(r=1, \ldots, s, j=1, \ldots, \hat{n})$. If $\mathbf{x}_{o} \leq \mathbf{0}$ put $\phi_{o}=1$ or 0 according as $\mathbf{y}_{o} \geq \mathbf{0}$ or $\mathbf{y}_{o}<\mathbf{0}$. Otherwise, for $o=1, \ldots, n$, choose $\phi_{o}, \lambda_{1 o}, \ldots, \lambda_{n o}$, $\hat{\lambda}_{1 o}, \ldots, \hat{\lambda}_{\hat{n} o}$ to

minimise $\phi_{o}$ subject to

$$
\begin{aligned}
& \sum_{j=1}^{n} y_{r j} \lambda_{j o}+\sum_{j=1}^{\hat{n}} \hat{y}_{r j} \hat{\lambda}_{j o} \geq y_{r o}, \quad(r=1, \ldots, s) \\
& \sum_{j=1}^{n} x_{i j} \lambda_{j o}+\sum_{j=1}^{\hat{n}} \hat{x}_{i j} \hat{\lambda}_{j o} \leq x_{i o} \phi_{o}, \quad(i=1, \ldots, m) \\
& \sum_{j=1}^{n} \lambda_{j o}+\sum_{j=1}^{\hat{n}} \hat{\lambda}_{j o} \leq 1
\end{aligned}
$$

and

$$
\lambda_{j o} \geq 0 \quad(j=1, \ldots, n), \quad \hat{\lambda}_{j o} \geq 0 \quad(j=1, \ldots, \hat{n}) .
$$


We call this model the expanded (complete NRS) model.

If $\Psi_{\mathrm{c}}, \Psi_{\mathrm{e}}$, and $\Psi$ are the production possibility sets of the complete, expanded and diversification-consistent models then we have $\Psi_{\mathrm{c}} \subseteq \Psi_{\mathrm{e}} \subseteq \Psi$ because each notional fund must be contained in $\Psi$ but need not be contained in $\Psi_{\mathrm{c}}$. So if we can find notional funds in $\Psi \backslash \Psi_{\mathrm{c}}$ we can use them to construct an expanded model that better estimates the efficiencies of the diversificationconsistent model.

Figure 2 about here.

Figure 2 describes an iterative procedure that constructs successively better approximations to the diversification-consistent model. In this procedure $F$ represents the original funds, $\hat{F}$ the notional funds and $P$ the pairs of funds that have not yet been considered for some improving solution. Initially we impose no extra stopping condition. Section 5 shows what we might use in practice. At each iteration $\hat{F}$ may change and we consider a fund $e$ efficient or inefficient according as $\phi_{e}=1$ or not in the expanded model $((16)-(19))$ with $\left\{\hat{f}_{1}, \ldots, \hat{f}_{\hat{n}}\right\}=\hat{F}$.

The following observation shows why we need only consider $0.5(f+g)$ in the iterative procedure. It is a standard result, which is easy to show holds for the complete and expanded models. Put $\hat{\mathbf{x}}_{j}=\left(\hat{x}_{1}, \ldots, \hat{x}_{m}\right)$ and $\hat{\mathbf{y}}_{j}=\left(\hat{y}_{1}, \ldots, \hat{y}_{s}\right)(j=1, \ldots, \hat{n})$. Suppose $\sum_{j=1}^{n} \lambda_{j o}\left(\mathbf{x}_{j}, \mathbf{y}_{j}\right)+\sum_{j=1}^{\hat{n}} \hat{\lambda}_{j o}\left(\hat{\mathbf{x}}_{j}, \hat{\mathbf{y}}_{j}\right)$ is on the efficient frontier. Then, $\lambda_{j o}=0$ unless $\left(\mathbf{x}_{j}, \mathbf{y}_{j}\right)$ is on the frontier $(j=1, \ldots, n)$ and $\hat{\lambda}_{j o}=0$ unless $\left(\hat{\mathbf{x}}_{j}, \hat{\mathbf{y}}_{j}\right)$ is on the frontier $(j=1, \ldots, \hat{n})$.

We want to choose $f$ and $g$ so that $h$ is reasonably likely to be added to $S$. To achieve this we maintain the pairs of funds $P$ as a priority queue. We expect more effieicent funds to be better choices for $f$ and $g$. And we expect inequalities (15) to get further from equality as the correlations between the funds get further from -1 . These suggest $\phi_{j}^{2} \phi_{k}^{2}\left(1-\rho\left(f_{j}, f_{k}\right)\right)$ should be a reasonable priority measure and we find it works well in practice.

Section 5 shows how the iterative procedure works in practice.

Convergence of the iterative procedure and properties of its solution

To see why we might reasonably expect the iterative procedure to give efficiencies that converge to the values in the diversification-consistent model, consider the following result.

Theorem 4. Suppose $\tilde{f}_{1}, \ldots, \tilde{f}_{m+s}$ are funds in an expanded model with convexityconsistent measures. Suppose also $\lambda_{1} \geq 0, \ldots, \lambda_{m+s} \geq 0$ satisfy $\sum_{j=1}^{m+s} \lambda_{j} \leq 1$. Then 
1. if $x_{i}\left(\sum_{j=1}^{m+s} \lambda_{j} \tilde{f}_{j}\right)<\sum_{j=1}^{m+s} \lambda_{j} x_{i}\left(\tilde{f}_{j}\right)$ for some $i \in\{1, \ldots, m\}, x_{i}\left(\sum_{j=1}^{m+s} \mu_{j} \tilde{f}_{j}\right)<$ $\sum_{j=1}^{m+s} \mu_{j} x_{i}\left(\tilde{f}_{j}\right)$ whenever $\mu_{j}>0(j=1, \ldots, n)$ and $\sum_{j=1}^{m+s} \mu_{j}=1$;

2. if $y_{r}\left(\sum_{j=1}^{m+s} \lambda_{j} \tilde{f}_{j}\right)>\sum_{j=1}^{m+s} \lambda_{j} y_{r}\left(\tilde{f}_{j}\right)$ for some $r \in\{1, \ldots, s\}, y_{r}\left(\sum_{j=1}^{m+s} \mu_{j} \tilde{f}_{j}\right)>$ $\sum_{j=1}^{m+s} \mu_{j} y_{r}\left(\tilde{f}_{j}\right)$ whenever $\mu_{j}>0(j=1, \ldots, n)$ and $\sum_{j=1}^{m+s} \mu_{j}=1$.

Proof. Put $\lambda=\min \left\{\lambda_{j} / \mu_{j}: j=1, \ldots, n\right\}$ and $\lambda_{j}>0$. Then $\lambda>0$ and $\mu_{j}-\lambda \lambda_{j} \geq 0$ for $j=1, \ldots, n$. If $x_{i}\left(\sum_{j=1}^{m+s} \lambda_{j} \tilde{f}_{j}\right)<\sum_{j=1}^{m+s} \lambda_{j} x_{i}\left(\tilde{f}_{j}\right)$ for some $i \in\{1, \ldots, m\}, \mu_{j}>0(j=1, \ldots, n)$ and $\sum_{j=1}^{m+s} \mu_{j}=1$ then

$$
\begin{aligned}
x_{i}\left(\sum_{j=1}^{m+s} \mu_{j} \tilde{f}_{j}\right) & =x_{i}\left(\lambda \sum_{j=1}^{m+s} \lambda_{j} \tilde{f}_{j}+\sum_{j=1}^{m+s}\left(\mu_{j}-\lambda \lambda_{j}\right) \tilde{f}_{j}\right) \\
& \leq \lambda x_{i}\left(\sum_{j=1}^{m+s} \lambda_{j} \tilde{f}_{j}\right)+\sum_{j=1}^{m+s}\left(\mu_{j}-\lambda \lambda_{j}\right) x_{i}\left(\tilde{f}_{j}\right) \\
& <\lambda \sum_{j=1}^{m+s} \lambda_{j} x_{i}\left(\tilde{f}_{j}\right)+\sum_{j=1}^{m+s}\left(\mu_{j}-\lambda \lambda_{j}\right) x_{i}\left(\tilde{f}_{j}\right) \\
& =\sum_{j=1}^{m+s} \mu_{j} x_{i}\left(\tilde{f}_{j}\right) .
\end{aligned}
$$

The second part follows by a similar argument.

Since the production possibility set $\Psi_{\mathrm{e}}$ of an expanded model with convexityconsistent measures is an unbounded convex polytope, the practical consequence of Theorem 4 is that we need only check one point in the interior of each facet on the frontier of $\Psi_{\mathrm{e}}$ to find a new notional fund or show none exists. For $m+s>2$ the iterative procedure of Figure 2 only checks points on the boundary of each facet. However, it is reasonable to expect that if the interior points of a facet give new notional funds, then so should the boundary.

Theorem 3 shows we can choose risk measures that are monotonic. We do not require this for convexity-consistent measures. The following result shows how monotonicity might be useful.

Theorem 5. Suppose $f_{p}$ and $f_{q}$ are funds in a diversification-consistent model with nonnegative convexity-consistent monotonic measures. Then $f_{p} \geq$ $f_{q} \Longrightarrow \phi_{p} \geq \phi_{q}$. 
Proof. Suppose $f_{p} \geq f_{q}$. If $x_{i}\left(f_{p}\right)=0$ for $i=1, \ldots, m$ then $\phi_{p}=1 \geq \phi_{q}$ and so the result holds. Otherwise suppose $x_{i}\left(f_{p}\right)>0$ for some $i$. Then, for $r=1, \ldots, s$,

$$
y_{r}\left(\sum_{j=1}^{n} \lambda_{j p} f_{j}\right) \geq y_{r}\left(f_{p}\right) \geq y_{r}\left(f_{q}\right) .
$$

Similarly, for $i=1, \ldots, m$,

$$
x_{i}\left(\sum_{j=1}^{n} \lambda_{j p} f_{j}\right) \leq x_{r}\left(f_{p}\right) \phi_{p} \leq x_{i}\left(f_{q}\right) \phi_{p} .
$$

So $\lambda_{j q}=\lambda_{j p},(j=1, \ldots, n), \phi_{q}=\phi_{p}$ satisfies constraints (12)-(13), (11) and (8) and so $\phi_{p} \geq \phi_{q}$ as required.

Theorem 5 shows we can construct DEA models consistent with a general stochastic ordering. We note that, for any stochastic ordering $\preccurlyeq$, we can replace monotonicity with the condition that $\kappa(g) \leq \kappa(f)$ for $f, g \in \mathcal{F}$ with $f \preccurlyeq g$ for each measure $\kappa$. So, for example, we can construct diversificationconsistent models that are consistent with second or third degree stochastic dominance (Levy, 1992), as Lozano and Gutiérrez (2008, 2007) do for simple DEA models with one risk and one return measure. Some caution is needed. The definitions of consistency with stochastic ordering (Ogryczak and Ruszczyński, 2002; Lozano and Gutiérrez, 2008) give implications of the form $f \preccurlyeq g \Longrightarrow$ some conditions on the measures $\Longrightarrow \phi_{f} \leq \phi_{g}$ while we typically want $\phi_{f} \leq \phi_{g} \Longrightarrow f \preccurlyeq g$. We may be able to do no better than choose measures that make this reverse implication reasonably likely.

\section{A practical illustration}

We show here that the procedure of Figure 2 works well in practice and show it converges reasonably quickly to good estimates of the efficiencies of the diversification-consistent model.

We need some data. We use 60 monthly returns from 30 hedge funds between 2000 and 2204, taken from Center for International Securities and Derivatives Markets (2010). The data set contains ten of each of three classes of fund. These are market neutral (MN), global macro (GM) and long/short equity (LS). Global macro funds usually adopt a riskier strategy than long/short or market neutral ones. So we expect diverse risk-return 
characteristics in different fund types. We also expect substantial asymmetry in the distributions of returns, justifying a model that incorporates more than mean and standard deviation. The columns on the right of Table 1 summarise the main features of the fund returns and indicate substantial departure from normality. Table 2 shows the correlations among six funds. These reflect the pattern in the 870 correlations between pairs of funds: at $5 \%$ significance level, 38 are significantly negatively correlated and only 137 significantly positively correlated.

We use $\mathrm{C}++$ code to maintain the data structures and implement the iterative procedure, and parallel CPLEX (Ilog, 2008) to solve the linear programmes. We use a $2.66 \mathrm{GHz}$ Intel Core 2 duo processor and a Gnu/Linux operating system. The iterative procedure can run indefinitely. So we introduce a stopping criterion. We check the average reduction in efficiency after a small fixed number of improving iterations and stop when this average reduction falls below a prespecified tolerance. In practice we find that 10 improving iterations and a tolerance level of $10^{-6}$ works well and gives a solution in under ten seconds for the examples we consider.

We consider two examples. Both use a commensurable set of convexityconsistent measures including only nonnegative risk measures. Our first example illustrates two things. First it shows the improvement available from diversification. Second, it shows that, even without a risk-free investment, the diversification-consistent model may easily find portfolios with positive return and zero measured risk. It uses the mean return of the 30 funds as a return measure and $\max \left(\mathrm{CVaR}_{0.4}, 0\right)$ as a risk measure. Figure 3 shows the production possibility set and efficient frontier from our approximation to a diversification-consistent NRS model. The dashed line shows the frontier from the conventional NRS model. There is clear evidence that the conventional model substantially overestimates the efficiency of many funds.

Figure 3 about here.

Our second example uses risk measures max $\left(\mathrm{CVaR}_{0.05}, 0\right)$, $\max \left(\mathrm{CVaR}_{0.1}, 0\right)$, $\max \left(\mathrm{CVaR}_{0.2}, 0\right)$ and $\max \left(s_{2}-\bar{y}, 0\right)$, where $s_{2}$ is the lower semideviation and $\bar{y}$ the mean, and return measures $\bar{y}$ and $-\mathrm{CVaR}_{0.9}$. (Ogryczak and Ruszczyński (2002) show $\max \left(s_{2}-\bar{y}, 0\right)$ is coherent and consistent with second degree stochastic dominance.) Six performance measures is typical for a DEA model of investment funds (Gregoriou et al., 2005a). They are enough to model most of the features of the data: much of the variance, skewness and kurtosis. They 
give $f_{p} \leq f_{q} \Longrightarrow \phi_{p} \leq \phi_{q}$ for $p, q \in\{1, \ldots, n\}$ in the diversification-consistent model.

Table 1 about here

Column NRS shows the efficiencies from the NRS model described at the end of Section 2. Column DC shows the efficiencies found by the iterative procedure of Figure 2, which approximate closely those of the diversificationconsistent model. Column RATIO shows the mean : standard deviation ratio for each fund. The diversification-consistent model has lower efficiencies than the NRS model for all but one fund and changes the rank order of the efficiencies. The rank order of the mean : standard deviation ratio shows greater difference, indicating the weakness of a measure that ignores the shape of funds' distributions.

The efficient notional funds we find are portfolios of six of the ten most efficient funds. Table 2 shows the correlations between these six funds. These are far from the perfect correlation needed for the NRS model.

Table 2 about here

\section{Discussion}

We have identified the returns to scale and measures needed for a DEA model of investment funds and shown how to handle scope for diversification. However, a number of issues remain. Although the procedure of Figure 2 works well in practice, there may be a much more efficient method to estimate a set of frontier portfolios. This would help for large data sets or (see below) if we want to repeat the procedure many thousands of times.

Like all applications of DEA to modelling funds we know of, ours finds efficiencies that describe past rather than predict future performance. We treat measures as fixed quantities rather than random variables. This issue needs further investigation. We suggest an approach based on the bootstrap, which Simar and Wilson (2000); Dyson and Shale (2010) discuss for general DEA models. The multiple observations for each fund allow another bootstrap approach and we have some promising preliminary results. We need to repeat the iterative procedure many times but find mean efficiencies and confidence intervals. We also find the bootstrap resolves a problem with deterministic DEA: the fund with highest mean return (GM2640) is given efficiency 1 no matter how great its risk measures are. 
Our data come from a time series. We have ignored autocorrelation, which may affect any estimate of future efficiency. We have also ignored the time horizon of investors (Galagadera and Silvapulle, 2002). Different funds may be at their most efficient at different time horizons, and both autocorrelation and time horizons could be investigated further.

\section{References}

Acerbi, C., 2007. Coherent measures of risk in everyday market practice. Quantitative Finance 7 (4), 359-364.

Acerbi, C., Tasche, D., 2002. On the coherence of expected shortfall. Journal of Banking and Finance 26, 1487-1503.

Alexander, C., 2001. Market Models. John Wiley \&6 Sons, Chichester.

Artzner, P., Delbaen, F., Eber, J. M., Heath, D., 1999. Coherent measures of risk. Mathematical Finance 9, 203-228.

Banker, R. D., Charnes, A., Cooper, W. W., 1984. Some models for estimating technical and scale efficiencies in data envelopment analysis. Management Science 30, 1078-1092.

Basso, A., Funari, S., 2001. A data envelopment analysis approach to measure the mutual fund performance. European Journal of Operational Research $135,477-492$.

Bogetoft, P., 1996. DEA on relaxed convexity assumptions. Management Science 42 (3), 457-465.

Briec, W., Kerstens, K., 2009. Multi-horizon Markowitz portfolio performance appraisals: A general approach. Omega 37, 50-62.

Briec, W., Kerstens, K., 2010. Portfolio selection in multidimensional and partial moment space. Journal of Economic Dynamics \& Control 34, 636656.

Briec, W., Kerstens, K., Lesourd, J. B., 2004. Single-period Markowitz portfolio, performance guaging, and duality: a variation on the Luenberger shortage function. Jounal of Optimization Theory and Applications 120, $1-27$. 
Cazals, C., Florens, J.-P., Simar, L., 2002. Nonparametric frontier estimation: a robust approach. Journal of Econometrics 106, 1-25.

Center for International Securities and Derivatives Markets, 2010. Accessed March 2010.

URL http://www. isenberg. umass . edu/CISDM/

Charnes, A., Cooper, W. W., Rhodes, E., 1978. Measuring the efficiency of decision making units. European Journal of Operational Research 2, 429-444.

Chen, Z., Lin, R., 2006. Mutual fund performance evaluation using data envelopment analysis with new risk measures. OR Spectrum 28, 375-398.

Cooper, W. W., Seiford, L. M., Tone, K., 2007. Data Envelopment Analysis: A Comprehensive Text with Models, Applications, References and DEASolver Software, 2nd Edition. Springer.

Dyson, R. G., Shale, E., 2010. Data envelopment analysis, operational research and uncertainty. Journal of the Operational Research Society 61 (1), 25-34.

Eling, M., 2006. Performance management of hedge funds using data envelopment analysis. Financial Markets and Portfolio Management 20 (4), 442-472.

Färe, R. Grosskopf, S., Lovell, C., 1994. Production Frontiers. Cambridge University Press.

Galagadera, U. A., Silvapulle, P., 2002. Australian mutual fund performance appraisal using data envelopment analysis. Managerial Finance 28, 60-73.

Gregoriou, G. N., Rouah, F., Satchell, S., Diz, F., 2005a. Simple and crossefficiency of CTAs using data envelopment analysis. The European Journal of Finance 11 (5), 393-409.

Gregoriou, G. N., Sedzro, K., Zhu, J., 2005b. Hedge fund performance appraisal using data envelopment analysis. European Journal of Operational Research 164 (4), 555-571. 
Gregoriou, G. N., Zhu, J., 2005. Evaluating Hedge Fund and CTA Performance: Data Envelopment Analysis Approach. John Wiley $\mathscr{6}$ Sons, Inc., Hoboken, New Jersey.

Ilog, 2008. CPLEX. http://www.ilog.com/products/cplex/, accessed July 2009.

Jorion, P., 2007. Value at Risk, 3rd Edition. McGraw-Hill.

Joro, T., Na, P., 2006. Portfolio performance evaluation in a mean-varianceskewness framework. European Journal of Operational Research 175, 446461.

Krause, A., 2002. Coherent risk measurement: an introduction. Balance Sheet 10, 13-17.

Levy, H., 1992. Stochastic dominance and expected utility. Management Science 38, 555-593.

Lhabitant, F.-S., 2004. Hedge Funds: Quantitative Insights. John Wiley $\&$ Sons, Chichester.

Lovell, C. A. K., Pastor, J. T., 1995. Units invariant and translation invariant DEA models. Operations Research Letters 18, 147-151.

Lozano, S., Gutiérrez, E., 2007. TSD-consistent performance assessment of mutual funds. Journal of the Operational Research Society 59, 1352-1362.

Lozano, S., Gutiérrez, E., 2008. Data envelopment analysis of mutual funds based on second-order stochastic dominance. European Journal of Operational Research 189, 230-244.

Morey, M. R., Morey, R. C., 1999. Mutual fund performance appraisals: a multi-horizon perspective with endogenous benchmarking. Omega 27, $241-288$.

Murthi, B. P. S., Choi, Y. K., Desai, P., 1997. Efficiency of mutual funds and portfolio performance measurement: A non-parametric approach. European Journal of Operational Research 98, 408-418.

Nguyen-Thi-Thanh, H., May 2006. On the use of data envelopment analysis in hedge fund selection, working Paper, Université d'Orléans. 
Ogryczak, W., Ruszczyński, A., 2002. Dual stochastic dominance and related mean-risk models. SIAM Journal on Optimization 13 (1), 60-78.

Russell, R. R., 1990. Continuity of measures of technical efficiency. Journal of Economic Theory 51, 255-267.

Scheel, H., Scholtes, S., 2003. Continuity of DEA efficiency measures. Operations Research 51 (1), 149-159.

Sengupta, J. K., 2003. Efficiency tests for mutual fund portfolios. Applied Financial Economics 13, 869-876.

Sharpe, W., 1966. Mutual fund performance. Journal of Business 39, 119138.

Shephard, R. W., 1970. Theory of Cost and Production Function. Princeton University Press, Princeton NJ.

Silva Portela, M. C. A., Thanassoulis, E., Simpson, G., 2004. Negative data in DEA: a directional distance approach applied to bank branches. Journal of the Operational Research Society 55, 1111-1121.

Simar, L., Wilson, P. W., 2000. Statistical inference in nonparametric frontier models: the state of the art. Journal of Productivity Analysis 13, 49-78.

Tone, K., 2001. A slacks-based measure of efficiency in data envelopment analysis. European Journal of Operational Research 130, 498-509.

Wilkens, K., Zhu, J., 2001. Portfolio evaluation and benchmark selection: a mathematical programming approach. Journal of Alternative Investments $4(1), 9-20$. 


\begin{tabular}{|c|c|c|c|c|c|c|c|c|}
\hline FUND & NRS & $\mathrm{DC}$ & RATIO & MEAN & $\mathrm{SD}$ & SKEW & KURT & $\overline{\mathrm{AC}}$ \\
\hline$\overline{\text { GM2640 }}$ & 1.000 & 1.000 & 0.239 & 0.022 & 0.094 & 0.487 & 1.982 & .364 \\
\hline LS90 & 1.000 & 0.912 & 0.420 & 0.020 & 0.048 & 0.458 & 1.641 & 0.211 \\
\hline GM2695 & 1.000 & 0.635 & 0.346 & 0.019 & 0.055 & 0.606 & -0.329 & 0.013 \\
\hline GM45 & 1.000 & 0.626 & 0.794 & 0.015 & 0.019 & 0.308 & -0.594 & 0.320 \\
\hline LS40 & 0.668 & 0.553 & 0.215 & 0.021 & 0.098 & 0.206 & 1.249 & 0.087 \\
\hline MN2681 & 0.535 & 0.247 & 0.648 & 0.014 & 0.022 & -0.214 & -0.241 & -0.121 \\
\hline GM 366 & 0.860 & 0.234 & 0.695 & 0.011 & 0.016 & 0.725 & 2.219 & 0.157 \\
\hline GM2693 & 0.881 & 0.231 & 0.700 & 0.011 & 0.016 & 0.703 & 2.170 & 0.166 \\
\hline MN629 & 0.720 & 0.186 & 0.673 & 0.008 & 0.012 & 0.053 & 0.899 & 0.310 \\
\hline MN2540 & 0.618 & 0.182 & 0.589 & 0.009 & 0.015 & 0.455 & 0.872 & 0.393 \\
\hline MN2547 & 0.470 & 0.178 & 0.545 & 0.007 & 0.013 & 0.690 & 1.870 & 0.043 \\
\hline MN2704 & 0.262 & 0.102 & 0.315 & 0.004 & 0.011 & 1.094 & 2.155 & 0.330 \\
\hline GM2889 & 0.315 & 0.088 & 0.419 & 0.010 & 0.024 & 0.000 & 1.624 & 0.066 \\
\hline $\operatorname{LS} 78$ & 0.171 & 0.065 & 0.250 & 0.007 & 0.028 & 0.192 & 0.379 & 0.098 \\
\hline LS 57 & 0.141 & 0.058 & 0.219 & 0.008 & 0.037 & 0.001 & -0.646 & -0.221 \\
\hline MN299 & 0.131 & 0.045 & 0.180 & 0.005 & 0.028 & 2.786 & 16.492 & -0.306 \\
\hline LS 6 & 0.101 & 0.042 & 0.151 & 0.009 & 0.063 & 0.941 & 1.906 & -0.099 \\
\hline MN301 & 0.117 & 0.041 & 0.164 & 0.005 & 0.028 & 2.760 & 16.290 & -0.312 \\
\hline LS64 & 0.102 & 0.037 & 0.144 & 0.006 & 0.041 & 2.355 & 11.882 & -0.181 \\
\hline GM 6876 & 0.010 & 0.033 & 0.186 & 0.006 & 0.030 & -0.081 & 2.067 & 0.195 \\
\hline GM59 & 0.079 & 0.026 & 0.139 & 0.006 & 0.046 & 0.095 & 1.901 & 0.020 \\
\hline GM3434 & 0.069 & 0.024 & 0.108 & 0.006 & 0.056 & 1.469 & 7.007 & -0.099 \\
\hline LS32 & 0.046 & 0.019 & 0.070 & 0.003 & 0.037 & 1.752 & 7.259 & -0.140 \\
\hline LS 20 & 0.044 & 0.018 & 0.067 & 0.003 & 0.037 & 1.759 & 7.297 & -0.142 \\
\hline MN 147 & 0.043 & 0.017 & 0.085 & 0.001 & 0.015 & 0.139 & 1.268 & -0.072 \\
\hline LS 79 & 0.037 & 0.012 & 0.068 & 0.006 & 0.082 & -1.146 & 6.987 & -0.088 \\
\hline MN2639 & 0.027 & 0.010 & 0.053 & 0.001 & 0.020 & 0.063 & 1.378 & -0.168 \\
\hline GM2068 & 0.023 & 0.008 & 0.051 & 0.002 & 0.038 & -1.417 & 3.012 & 0.124 \\
\hline LS39 & 0.007 & 0.003 & 0.013 & 0.001 & 0.083 & 0.493 & 0.550 & 0.020 \\
\hline MN2777 & 0.000 & 0.000 & -0.018 & -0.001 & 0.044 & -0.381 & 0.297 & 0.010 \\
\hline
\end{tabular}

NRS: efficiency from NRS model; DC: efficiency from expanded model approximating diversification-consistent model; RATIO: mean-standard-deviation ratio; MEAN: mean return; SD: standard deviation in return; SKEW: skewness in return; KURT: kurtosis in return; AC: serial autocorrelation in return series. Boldface indicates differences from zero significant at $5 \%$ level.

Table 1: Efficiencies and summary statistics for 30 funds 


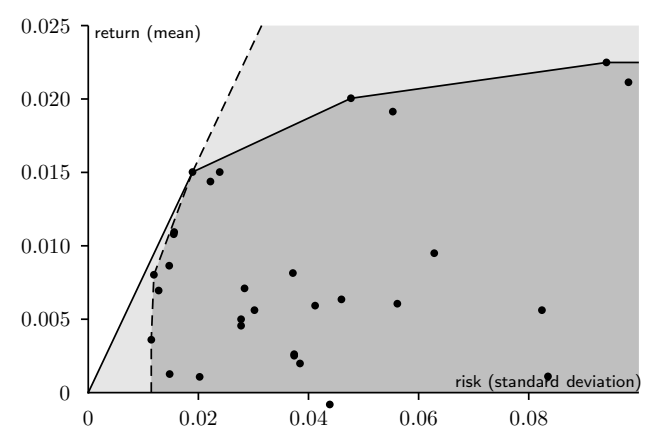

Figure 1: Comparison of different returns to scale

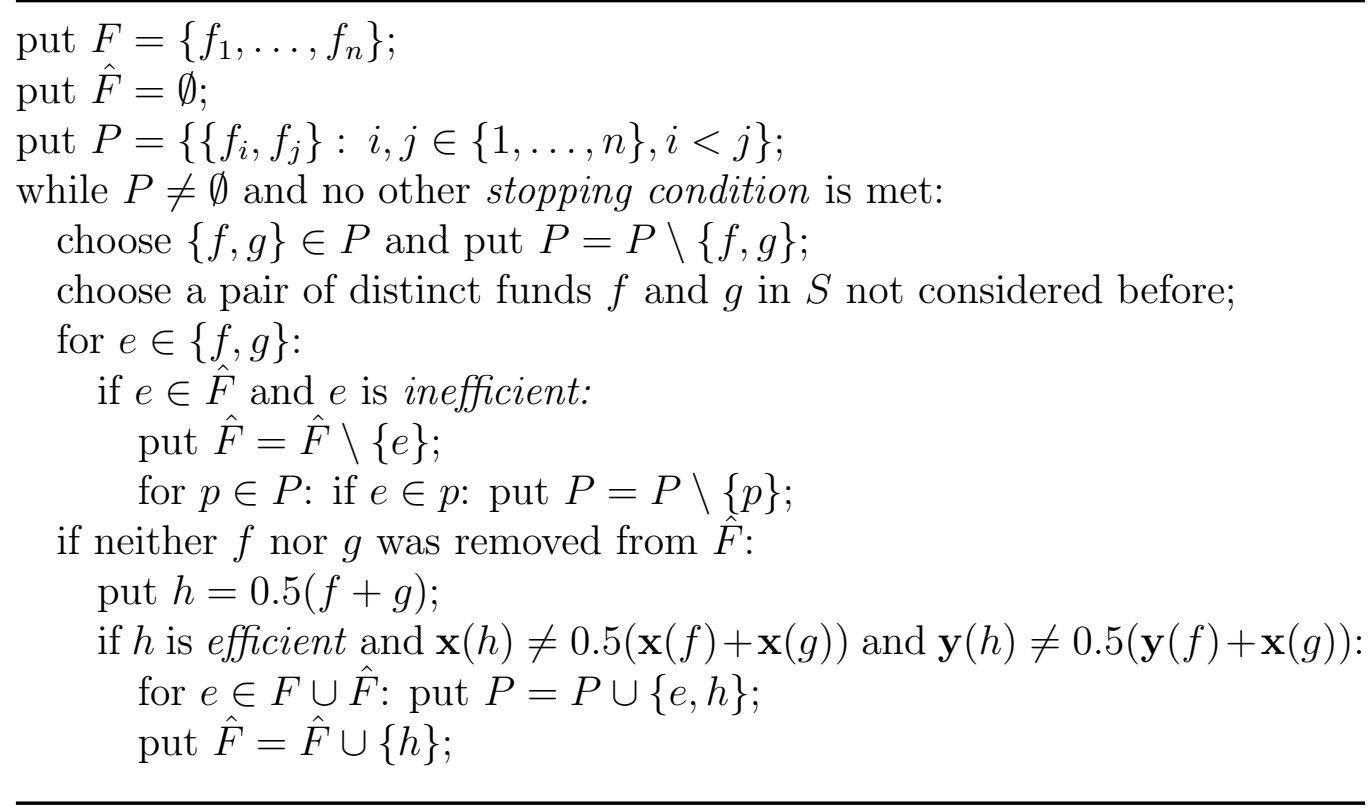

Figure 2: Iterative procedure to generate expanded models 


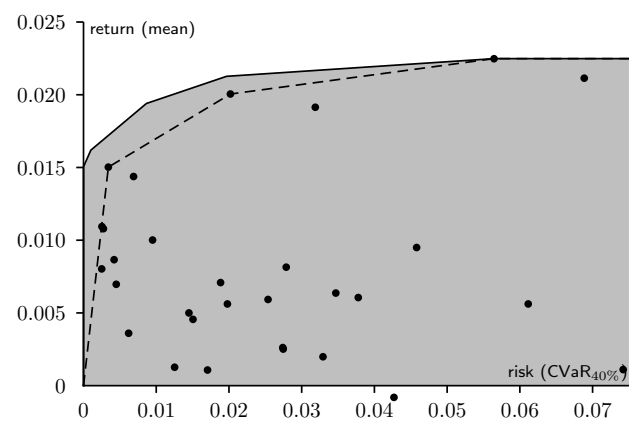

Figure 3: Approximating a diversification-consistent NRS model

\begin{tabular}{lrrrrr}
\hline FUND & LS90 & GM2695 & GM45 & GM2693 & MN2540 \\
\hline GM2640 & -0.144 & 0.303 & $-\mathbf{0 . 3 0 6}$ & -0.084 & -0.136 \\
LS90 & & 0.063 & 0.295 & 0.121 & 0.390 \\
GM2695 & & & 0.387 & 0.252 & -0.074 \\
GM45 & & & & 0.357 & 0.160 \\
GM2693 & & & & & 0.030 \\
\hline
\end{tabular}

Italics indicates correlation not significantly different from zero at $5 \%$ level; bold italics indicates correlation negative and significantly different from zero at $5 \%$ level.

Table 2: Correlation coefficients for six funds 